\title{
Establishing a new cardiac surgery service in an Australian university hospital: Pitfalls and lessons learned
}

Follow this and additional works at: https://www.journal.acorn.org.au/jpn

Part of the Health Services Administration Commons, Health Services Research Commons, Perioperative, Operating Room and Surgical Nursing Commons, and the Surgery Commons

(c) (i)

This work is licensed under a Creative Commons Attribution 4.0 License.

\section{Recommended Citation}

Ciobanu, Claudia; Latimer, Sharon; and Gillespie, Brigid M. (2018) "Establishing a new cardiac surgery service in an Australian university hospital: Pitfalls and lessons learned," Journal of Perioperative Nursing: Vol. 31 : Iss. 3 , Article 6.

Available at: https://doi.org/10.26550/2209-1092.1037

https://www.journal.acorn.org.au/jpn/vol31/iss3/6

This Article is brought to you for free and open access by Journal of Perioperative Nursing. It has been accepted for inclusion in Journal of Perioperative Nursing by an authorized editor of Journal of Perioperative Nursing. 


\author{
Authors \\ Claudia Ciobanu \\ M Hlth Serv Mgt \\ Surgical and Procedural Services, Gold \\ Coast Hospital and Health Service, \\ Queensland, Australia \\ HSM, School of Medicine, Griffith \\ University, Gold Coast, Queensland, \\ Australia
}

Dr Sharon Latimer

PhD, RN

School of Nursing and Midwifery, Griffith

University, Gold Coast, Queensland,

Australia

Nursing/Midwifery Education and

Research Unit, Gold Coast Hospital and

Health Service, Queensland, Australia

Professor Brigid M Gillespie

PhD, RN, FACORN

School of Nursing and Midwifery, Griffith University, Gold Coast, Queensland,

Australia

Gold Coast Hospital and Health Service, Queensland, Australia

\section{Corresponding author}

Claudia Ciobanu

M Hlth Serv Mgt

Surgical and Procedural Services, Gold

Coast Hospital and Health Service,

Queensland, Australia

HSM, School of Medicine, Griffith

University, Gold Coast, Queensland,

Australia

claudia.ciobanu@griffithuni.edu.au

\section{Authors' contributions}

BMG and SL co-supervised CC in writing up this project. CC drafted the manuscript; BMG and SL edited and contributed to manuscript writing for important intellectual content. CC, BMG and SL contributed to interpretation and approved the manuscript.

\title{
Establishing a new cardiac surgery service in an Australian university hospital: Pitfalls and lessons learned
}

Using change management to implement a new surgical service

\begin{abstract}
Cardiovascular disease is the leading cause of death among Australians. Creating and delivering a world-class cardiac surgery service in a cost-effective way is the principle goal of health care organisations and managers. Establishing a new health care service is a unique opportunity and requires detailed planning and implementation. Managing change is pivotal to establishing a new patient-focused health care service, and should be coupled with effective communication and teamwork among all stakeholders. There are foreseen and unforeseen challenges associated with any project so sharing the lessons learned is an important way for others in a similar situation to avoid potential pitfalls.
\end{abstract}

Keywords: health services, change management, cardiac surgery, change theories, nursing process, operating room

\section{Introduction}

Cardiovascular disease is the principle cause of death across Australia and worldwide1. In Australia, cardiovascular disease affects more than three million people, with individuals living in regional and rural areas more likely to succumb to this disorder compared to those in metropolitan areas ${ }^{2}$. Creating an accredited cardiac surgery service in a regional area increases the equity of health care access and reflects the National Strategic Framework for Rural and Remote Health ${ }^{3}$. To date, cardiac surgery remains the best treatment for those patients with complex cardiovascular disease ${ }^{4}$.

Establishing a new cardiac surgery service across a large regional community is complex and requires detailed planning and organisation. The overall aim is to deliver a high quality, evidence-based cardiac surgery service in a cost-effective, safe and efficient manner ${ }^{5}$. Based on the literature and situated in the public hospital context, this article outlines the process of establishing a cardiac operating room (OR) in a new adult cardiac surgery service at the Gold Coast University Hospital (GCUH). Also outlined are the challenges and lessons learned during this process.

Cardiac surgery services are a highly specialised area of health care involving the diagnosis, management and treatment of patients with an extensive range of cardiac diseases such as severe coronary artery disease ${ }^{3}$. Following referral to these services, patients can receive a range of highly skilled interventions and treatments such as coronary bypass grafts or valve surgery ${ }^{3}$. Delivered by a specialised multi-disciplinary cardiac surgical team in the OR, the patient's continuity of care spans 
across the perioperative and postoperative clinical areas. Broadly speaking, cardiac surgery services encompass a cardiac surgical ward, a coronary care unit (CCU), the intensive care unit (ICU) and the cardiac OR ${ }^{6}$. Considered the centre of cardiac surgery services, the cardiac $\mathrm{OR}$ is the focal point of all elective and emergency cardiac surgeries. The key clinical performance indicators of the cardiac OR team are measured in terms of complications and mortality rates, and determine the achievements of any cardiac surgery facility

The GCUH opened in 2013 and services the population across the Gold Coast district, which extends from south of Logan River to Tweed Heads on the Queensland (QLD)New South Wales (NSW) border, and west to Beechmont and Tamborine Mountain. GCUH also accepts patients from northern NSW. Prior to the establishment of the new cardiac surgery service, patients from the Gold Coast region who required cardiac surgery were transferred to Brisbane hospitals, more than 70 kilometres away. More than 300 Gold Coast patients were travelling to Brisbane each year for cardiac surgery $^{8}$. Due to the predicted population growth across the Gold Coast and northern NSW regions, coupled with the high number of tourists ${ }^{9}$, it was determined that the ability to provide cardiac surgery to 400 patients a year was required to meet the region's growing health care needs. Planning of the new cardiac surgery service at GCUH commenced in 2013, with the first cardiac surgery performed on 19 February 2014. Establishing a new health care service is a major undertaking so consulting the current evidence is an important first step.

\section{Literature review}

To set up a new clinical service, a number of well thought out steps and detailed planning are required; these include implementation of change management theories, access to adequate resources (specialised staff, economic resources and equipment), an appropriate timeline, adequate evaluation and identification of barriers and enablers. In addition, the new cardiac surgery service should be evidence-based and reflect current clinical standards and guidelines ${ }^{10}$. A review of the literature and current Australian and New Zealand Society of Cardiac and Thoracic Surgeons guidelines for establishing an adult cardiac surgery unit are presented ${ }^{10}$.

Cardiac surgery is a relatively new specialty that, with the advancement of technology, is rapidly evolving. For example, the first heart transplant was performed in 1967, only three years after the first effective coronary artery bypass grafting ${ }^{11}$. Since then, many changes have been implemented in cardiac surgery. Most importantly, the development of advanced cardiopulmonary life support improved the safety of heart surgery, offering surgeons the opportunity to perform complex surgical procedures on high-risk patients, with good patient outcomes reported ${ }^{11}$. The ongoing and extensive testing and improvements associated with cardiac surgery has improved cardiovascular mortality rates ${ }^{12}$. This demonstrates the fast rate of change in this clinical specialty so having a detailed understanding of the change management process in health care is a key to establishing a new cardiac surgery service.

\section{Change management theories to guide implementation}

Change within any organisation is challenging for staff and the organisation ${ }^{13}$; however, with mutual support and co-operation the strategic goals can be achieved ${ }^{14}$. Allen ${ }^{13}$ p. 58 stated that "change is a challenge that most organisations experience' and the capability of change, adjustment and progress is the benefit of this process ${ }^{15}$. The implementation of change needs to be effective to guarantee positive outcomes for patients and health care staff ${ }^{16}$. It should involve developing efficient implementation strategies, which include every stakeholder, and be well coordinated and flexible enough to anticipate and respond to any issues that may disrupt the change process ${ }^{16}$. Two major organisational change theories were used to guide the establishment of the new cardiac OR at GCUH. These were Lewin's theory (1951) which identifies three stages of change process, and Lippitt's theory (1958) which presents seven phases of the change management process ${ }^{14}$.

Kurt Lewin was a pioneer in change theories $^{14}$ and, in 1951, outlined three stages during the change process:

1. unfreezing, which involves assessing the current situation and planning for the upcoming change $^{14}$

2. changing or moving, which involves the implementation of the proposed changes

3. refreezing, which encompasses the evaluation and consolidation of the changes ${ }^{17}$.

Lewin's theory is considered the most effective for the change and implementation process and has been widely used in health care change processes, nursing research, professional nursing practice, nursing education and management ${ }^{18}$. In Lewin's theory, the implementation process should commence only once the first stage of unfreezing ends, thus ensuring a viable strategic 
plan and readiness of all staff and stakeholders $^{18}$. According to Lewin's theory, during the final refreezing stage it is vital to guarantee that the change is sustainable, including stabilising the change and safeguarding the efforts towards and commitment to the change ${ }^{13}$.

Meanwhile, an extension of Lewin's theory proposed by Ronald Lippit in 1958, has seven phases ${ }^{14}$. These seven phases include:

1. identifying the need for change

2. evaluating the drive for and ability to change

3. considering the change agent's drive and resources

4. selecting a clear change objective

5. indicating suitable roles for the agents involved

6. sustaining the change

7. concluding the liaison with those assisting $^{14}$.

The phases in Lippitt's theory are comparable to the components of the nursing process - assessment, planning, implementation and evaluation - making the theory easy to follow and providing a comprehensive implementation plan specific to health care settings ${ }^{14}$. The four steps of the nursing process and Lippitt's theory are fundamentally connected. For example, the assessment phase in the nursing process is similar to the first three phases of Lippitt's theory (identifying, evaluating and considering $)^{14}$. Planning in the nursing process corresponds to Lippitt's fourth and fifth phases (selecting and indicating) ${ }^{14}$. Lippitt's sixth phase (sustaining) is similar to implementation in the nursing process, while phase seven (concluding) mirrors evaluation ${ }^{14}$.

An essential difference between the two theories is that Lippitt focuses more on the change agent, provides more time to implement change and involves frequent assessment of the changing process; thus increasing the confidence of health care staff management and confirming the selection of this theory in health care change management ${ }^{17}$. Alternatively, Lewin's three-stage theory is more suited to moderate changes, implemented in constant circumstances $^{13}$. A comparison between the two theories and the nursing process is presented in Table 1.

\section{Human and material resources}

Cardiac surgery is a resourceintensive and costly component of a hospital budget that needs specialised staff and equipment ${ }^{10,19}$. A clear vision and ongoing financial support, underpinned by sound decision-making processes are needed to make the unit feasible in the long term and meet budgetary constraints? The cardiac OR is complex ${ }^{20}$ - patient care is provided by a recognised and specially qualified interdisciplinary clinical team ${ }^{6,10}$. Human resources are pivotal to the delivery of cardiac surgery services, with the team comprising cardiac surgeons, perioperative nurses, anaesthetists and clinical perfusionists ${ }^{5}$. Cardiac team members need highly specialised skills ${ }^{20}$ and the ability to manage highly technical equipment ${ }^{5}$.

According to current ANZSCTS guidelines $^{10}$, a new cardiac service should be situated in a tertiary hospital ${ }^{5,10}$ with the necessary staff

Table 1. Lewin's and Lippitt's theories compared in association with nursing process (Adapted from Mitchell 2013)

\begin{tabular}{|c|c|c|c|c|}
\hline Nursing process & \multicolumn{2}{|c|}{ Lippitt's theory } & \multicolumn{2}{|c|}{ Lewin's theory } \\
\hline \multirow[t]{3}{*}{ Assessment } & Phase 1 & Identify the need for change & \multirow[t]{3}{*}{ Unfreezing } & \multirow{3}{*}{$\begin{array}{l}\text { Study the situation and } \\
\text { increase motivation for change }\end{array}$} \\
\hline & Phase 2 & $\begin{array}{l}\text { Evaluate drive for and ability to } \\
\text { change }\end{array}$ & & \\
\hline & Phase 3 & $\begin{array}{l}\text { Consider change agent's drive and } \\
\text { resources }\end{array}$ & & \\
\hline \multirow[t]{2}{*}{ Planning } & Phase 4 & Select clear change objective & \multirow[t]{3}{*}{ Moving } & \multirow{3}{*}{$\begin{array}{l}\text { Implement changes, involve } \\
\text { people }\end{array}$} \\
\hline & Phase 5 & $\begin{array}{l}\text { Indicate suitable roles for agents } \\
\text { involved }\end{array}$ & & \\
\hline Implementation & Phase 6 & Sustain change & & \\
\hline Evaluation & Phase 7 & $\begin{array}{l}\text { Conclude the liaison with those } \\
\text { assisting }\end{array}$ & Refreezing & $\begin{array}{l}\text { Consolidate changes and } \\
\text { provide positive reinforcement } \\
\text { of desired results }\end{array}$ \\
\hline
\end{tabular}


and facilities to support safe patient care before and after cardiac surgery ${ }^{6}$. Importantly, this new service should also have an established referring relationship with a reputable cardiac surgical unit that has been accredited for at least three years ${ }^{5,10}$.

Reflecting current guidelines, a cardiac surgery service requires the following facilities, including the cardiac OR 5,10 .

- at least one operating room dedicated to cardiac surgery

- an ICU with adequate equipment for patient care after cardiac surgery

- a cardiac catheterisation and angiography laboratory (It is highly recommended that a hybrid suite is available or the possibility to upgrade exists.)

- a distinct cardiac surgical ward, with dedicated patient monitoring systems and telemetry, to treat patients pre- and post-operatively

- a high dependency or step-down unit well equipped to be able to care for high risk cardiac and thoracic patients

- a radiology department that provides portable $\mathrm{X}$-rays, image intensifier services, CT scans and cardiac MRIs

- an echocardiography unit, with echo apparatus with transoesophageal echocardiogram (TOE) and 3-D capability, for every cardiac theatre and a transportable machine available for patients in ICU or other units

- a well-resourced blood bank; pharmacy, pathology and haematological facilities; and a microbiology and biochemistry test centre.

These facilities should be located close to and with direct access from the cardiac OR, guaranteeing ease of communication and patient transfer without functional impediments in patient care ${ }^{7,10}$.

In addition, the hospital should have allied health services, including physiotherapists, dietitians, rehabilitation and occupational therapists, to aid in the patient's post-operative recovery and reduce complications ${ }^{21}$. At the same time, the patient's continuity of care is supported by access to other services ${ }^{22}$, such as an infectious disease service, infection control policies, a respiratory unit, pacemaker installation facilities, nuclear medicine $e^{5,10}$ and patient counselling and education services. During the immediate post-operative period, an on-call cardiopulmonary resuscitation team should be available to respond to any postoperative emergency.

Furthermore, the development and implementation of new health care service processes, policies and procedures are fundamental to the delivery of quality and safe patient care. It is very important to develop an appropriate and agreed timeline for planning and implementing these processes, thus providing staff with an element of certainty ${ }^{7,13}$. Regular review of established timelines is also necessary so that responsive changes can be made and the project outcomes met ${ }^{13}$.

\section{Evaluation}

Ongoing evaluation is an integral component of change management. Although frequently viewed as an important final stage, it should include enquiries about the achievements and sustainability of the change and the completion or need for additional action ${ }^{13}$. This should be followed by quality reflection that will confirm the project outcomes ${ }^{23}$. Evaluation can sometimes result in the revisiting of earlier stages in the change management process, and the implementation of new actions ${ }^{13}$. These actions should be viewed as a normal part of the change process, not as an indication of failure ${ }^{13}$.

The implementation of change can face numerous obstructions to achieving the desired outcomes; however, a cautious selection and use of a change management process theory can prevent many obstacles from impeding the chance of success ${ }^{14}$. Changes in health care are at risk of collapse due to barriers such as poor planning, a lack of qualified staff, inadequate structural organisation and poor economic support or lack of commitment ${ }^{17}$. Similarly, the success of a new health care service can be influenced by enablers such as good leadership and planning, good communication and team commitment with organisational support ${ }^{14}$. Finally, the context within which the change is implemented is also considered an important factor influencing the project's success ${ }^{17}$.

\section{Establishing the new cardiac surgery service at GCUH}

The GCUH opened on a new site in September 2013, replacing the old Gold Coast Hospital campus at Southport. The GCUH is one of the largest clinical training and research facilities in Queensland, Australia, and includes a busy emergency department. The hospital has been future-proofed and allows for the increasing health needs of the fastgrowing population of the Gold Coast region. The region has a population of 560000 people and receives approximately 13 million tourists each year ${ }^{24}$. The hospital is a tertiarylevel facility that offers modern, high quality hospital care and includes 750 beds and has over 8900 employees $^{25}$. It is co-located with the Gold Coast Private Hospital and the Gold Coast Health and Knowledge 
Precinct. The health services delivered at the GCUH include the categories of women and children, care and support, immunisation, emergency and trauma, maternity, medical and surgical, mental health, oral health and public health ${ }^{25}$. Over 13200 elective surgeries and 6700 emergency surgeries, including 345 cardiac surgeries, were performed in the OR of GCUH in the 2016-2017 financial year ${ }^{24}$, demonstrating the high patient acuity and activity levels.

The extensive process of setting up the new cardiac ORs was challenging with some barriers encountered along the way. The fundamental focus was patient safety and the quality of care. The OR department at GCUH comprises 20 operating suites, with 18 fully commissioned and two well-equipped cardiac rooms. Approximately 190 perioperative nurses currently work in the entire OR department. The cardiac OR is staffed by nine cardiac scrub/scout and nine cardiac anaesthetic registered nurses. The cardiac perioperative team also includes three surgeons and seven anaesthetists and perfusionists.

Table 2 (on the next page) outlines the process of setting up the new cardiac OR at GCUH based on the ANZSCTS guidelines ${ }^{10}$ and correlated with the phases of Lippitt's theory ${ }^{14}$.

For a successful implementation of the new cardiac OR, a significant continuing commitment of time and determination from the entire team is essential to ensure a culture of quality development by observing and matching the standards of care delivered ${ }^{26}$. At the same time, this will improve perioperative competencies, work arrangement and ability to manage change ${ }^{15}$. Achieving the identified objectives of establishing a new health care service is invariably challenged by different barriers.

\section{Barriers and enablers to setting up a new cardiac operating room}

An effective implementation of a new health care service includes the management of inevitable barriers that can stall the process ${ }^{13}$. Having an efficient plan, understanding the change management theories and recognising the possible barriers to the process are ways to ensure success ${ }^{17}$. The barriers identified through the process of establishing the new cardiac surgery service at GCUH are related to human resources, structural and financial factors.

\section{Human resources barriers}

Human resources, together with physical resources and consumables, are the main requirements needed for a health care system to deliver high quality care so effective management of human resources is important ${ }^{27}$. Four specialised disciplines make up the cardiac surgical team, which is led by the surgeon who works in synchronicity with the anaesthetist, scrub/scout and anaesthetic nurses and the perfusionist. The role of each team member is clearly defined and effective teamwork, characterised by continuous synchronisation of responsibilities, contributes to maintaining safe surgical practice. With scrupulous observation and meticulous attention to detail team members monitor the progress of the surgery and anticipate the next step or any potential adverse event ${ }^{28}$.

Patient outcomes from cardiac surgery can be compromised by a lack of appropriately skilled multidisciplinary staff. This challenge is compounded by the global shortage of specialised medical and nursing staff 7 . Evidence suggests nursing staff who specialise in cardiac surgery can result in reduced rates of adverse events ${ }^{29}$. The difficulty of recruiting specialised cardiac surgical nurses was acknowledged as a major challenge by GCUH from the outset because of the shortage of these highly trained nurses in the Gold Coast region. The first step to overcoming this barrier to an effective OR was identifying capable nursing staff already working in the OR at GCUH and seconding them to established cardiac surgery services for extensive training. The other strategy was to actively recruit experienced cardiac surgical nurses from across Australia. Both strategies required time and money to implement and organisational support was an important factor. To increase sustainability and staff retention, ongoing targeted education and in-service sessions were established along with set-up exercises and mock trials.

When the first cardiac surgery was performed at the GCUH, the cardiac OR had only three experienced cardiac instrument/ circulating registered nurses and four anaesthetic registered nurses. These numbers were insufficient to maintain the service or meet the needs of the service's proposed elective and emergency activity. Clearly, a proactive approach to expansion and succession planning was required to increase the number of suitably experienced staff. One strategy to address this in the short term was to train experienced perioperative nurses from other specialties, such as vascular or general surgery, who expressed an interest in joining the cardiac team.

\section{Structural barriers}

A new cardiac surgery service requires organised space for the proposed activities and storage areas for equipment. According to ANZSCTS guidelines ${ }^{10}$, the cardiac ICU and the cardiac surgical ward should be in close proximity to the cardiac 
Table 2: Outline of setting up the new cardiac OR at GCUH correlated with the phases of Lippitt's theory

\begin{tabular}{|c|c|}
\hline $\begin{array}{l}\text { Phase 1: } \\
\text { Identifying } \\
\text { the need for } \\
\text { change }\end{array}$ & $\begin{array}{l}\text { The new cardiac OR was to be part of GCUH's cardiac service level } 6 \text { that provides complete surgical cardiac care, } \\
\text { including composite multisystem life support to critically ill patients. } \\
\text { The service was proposed to be a networked adult cardiac service to provide a comprehensive range of cardiac } \\
\text { care services with certain exclusions such as transplantation and paediatric cardiac surgery and to achieve } \\
\text { about } 400 \text { cases per annum?. } \\
\text { The service would include onsite access to balloon pump insertion and management, onsite access to cardiac } \\
\text { intensive care for pre- and post-operative care and an interventional cardiac service9. } \\
\text { The service should include a perioperative cardiac service level } 6 \text { along with all other services at the same level, } \\
\text { including: } \\
\text { - cardiac medicine } \\
\text { - cCU } \\
\text { - cardiac diagnostics and intervention } \\
\text { - cardiac rehabilitation unit } \\
\text { - cardiac ICU } \\
\text { - cardiac anaesthetics } \\
\text { - pathology and pharmacy. }\end{array}$ \\
\hline $\begin{array}{l}\text { Phase 2: } \\
\text { Evaluating } \\
\text { the drive for } \\
\text { and ability to } \\
\text { change }\end{array}$ & $\begin{array}{l}\text { The incentive for change was the desire for a new cardiac OR that would provide high quality surgical care to } \\
\text { cardiac patients. Key deliverables related to this were identified as: } \\
\text { - patient-centred model of care and service ramp-up plan } \\
\text { - equipment and instrumentation } \\
\text { - consumables and consignment stock } \\
\text { - support service prepared } \\
\text { - service evaluation plan } \\
\text { - emergency response. } \\
\text { Indicators of the ability to make the change included: } \\
\text { - a service agreement with private sector } \\
\text { - a workforce plan including staff recruitment and education } \\
\text { - policies, procedures and protocols } \\
\text { - a communication plan. }\end{array}$ \\
\hline $\begin{array}{l}\text { Phase 3: } \\
\text { Considering } \\
\text { the change } \\
\text { agent's } \\
\text { drive and } \\
\text { resources }\end{array}$ & $\begin{array}{l}\text { Consideration of financial and human resources included: } \\
\text { - a budget and activity plan } \\
\text { - benchmarking with the established cardiac services and local private providers } \\
\text { - a stakeholder and staffing profile of cardiac operating room teams - surgeons, nurses, anaesthetic doctors, } \\
\text { anaesthetic nurses and medical perfusionists } \\
\text { - the nursing team: nursing staff responsible for direct patient care are registered nurses, with enrolled nurses, } \\
\text { student nurses and assistants in nursing with the support and supervision of registered nurses } \\
\text { - at least one registered nurse competent in providing advanced life support rostered on at all times } \\
\text { - qualifications of nursing team: all nursing staff with or working towards relevant competencies and education } \\
\text { programs in cardiac perioperative nursing } \\
\text { - two categories of nursing staff - cardiac scrub/scout nurses and cardiac anaesthetic nurses. }\end{array}$ \\
\hline
\end{tabular}




\begin{tabular}{|c|c|}
\hline $\begin{array}{l}\text { Phase 4: } \\
\text { Selecting a } \\
\text { clear change } \\
\text { objective }\end{array}$ & $\begin{array}{l}\text { The objective was to implement cardiac surgery in a safe and timely manner through: } \\
\text { - a patient-centred model of care with a focus on quality } \\
\text { - confirming stability of care across the service providers } \\
\text { - meeting activity targets (GCHHS service agreement 2013) } \\
\text { - utilising available funds in the most efficient way possible to deliver high quality care. }\end{array}$ \\
\hline $\begin{array}{l}\text { Phase 5: } \\
\text { Indicating } \\
\text { suitable } \\
\text { roles for } \\
\text { the agents } \\
\text { involved }\end{array}$ & 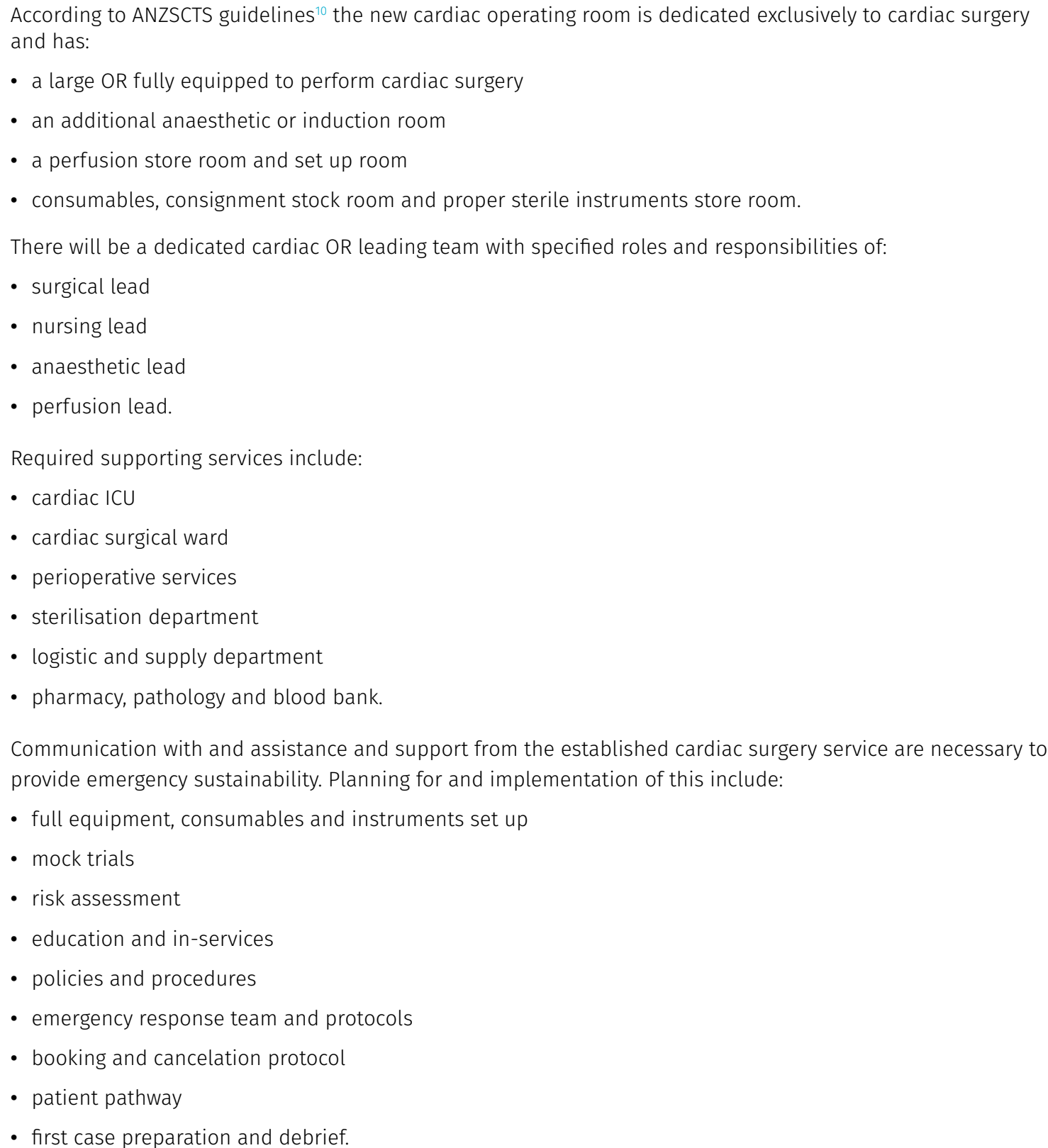 \\
\hline
\end{tabular}


Table 2 (continued): Outline of setting up the new cardiac OR at GCUH correlated with the phases of Lippitt's theory

\begin{tabular}{|c|c|}
\hline $\begin{array}{l}\text { Phase 6: } \\
\text { Sustaining } \\
\text { the change }\end{array}$ & $\begin{array}{l}\text { The new cardiac OR will be supported to meet the objective of providing cardiac surgery in a safe and timely } \\
\text { manner through: } \\
\text { - following policies and procedures } \\
\text { - implementing and improving emergency response (a 24-hour on-call cardiac surgery team) } \\
\text { - improving quality of care } \\
\text { - briefing and debriefing team meetings } \\
\text { - demonstrating processes for the formal audits and review of cardiac OR activity, quality and safety meetings, } \\
\text { mortality and morbidity meetings } \\
\text { - operational team meetings and communication. }\end{array}$ \\
\hline $\begin{array}{l}\text { Phase 7: } \\
\text { Concluding } \\
\text { the liaison } \\
\text { with those } \\
\text { assisting }\end{array}$ & $\begin{array}{l}\text { Service agreements with the established cardiac surgery unit and private sector can be concluded with } \\
\text { establishment of: } \\
\text { - independent activity of the cardiac OR and } 24 / 7 \text { coverage of emergency } \\
\text { - planned activity and budget } \\
\text { - developed patient pathways. }\end{array}$ \\
\hline
\end{tabular}

OR, or at least on the same floor, to guarantee a timely and safe transfer of patients pre- and post-operatively. The current design at GCUH has the OR on level two of the building and the ICU and cardiac wards on level four. As cardiac surgery patients are highly dependent and sometimes very unstable, transferring them between two floors was a challenge for the team. Faced with this barrier, the goal was to identify strategies that would ensure safe transfer of patients. One strategy was to have a well-equipped transport trolley attached to the patient bed during transfer, improving the management of any adverse events during transfer. The anesthetic teams transferring patients were also given an emergency keycard for the lifts to avoid any delays. During set-up, comprehensive trials and simulations were carried out to familiarise the team with the new environment and equipment, thus ensuring timely and safe patient transfer. All possible events were discussed and emergency protocols were established. Since the first cardiac surgery case in 2014, the team has performed over 1100 cardiac surgeries with no patient safety incidents associated with patient transfer having been reported.

In addition to spacial design, the hospital infrastructure needs to be carefully planned, especially because the cardiac OR and all the other perioperative departments need to function interdependently to ensure smooth transitions in patient care? As Mehta and Bhatia state, all cardiac ORs should have a minimum of two mobile pendants with additional gas flow meters for the cardiopulmonary bypass machine and anaesthetic equipment. The cardiac OR at GCUH has three mobile pendants, and only two have the additional gas flow meters and connections required for the bypass and anaesthetic machines. The position of the three pendants and the layout of the OR with five-door access jeopardises the sterility of the surgical set-up. Different set-ups and arrangements were tested to find the best position for the OR equipment to preserve a sterile surgical setup. To comply with infection control standards, a surgical sterile set-up must be undertaken in an isolated area with minimum traffic ${ }^{30}$. The solution implemented by the team was for the sterile surgical set-up to be conducted on the right side of the operating table and moved into the required position on the left side as soon as the patient was prepped and draped. This is an example of how the cardiac instrument/circulating nurses developed 'workarounds' to manage these types of structural barriers without any compromise to patient care.

Finally, a lack of storage space for cardiac surgery equipment, instrumentation and consumables that meets Australian standards presented another structural barrier to an effective OR. The Australian standards ${ }^{10}$ recommend that all equipment and instrumentation be in close proximity of the cardiac OR for easy access in an emergency. The space allocated for cardiac surgery consumables and instrument storage was limited in the department at GCUH. The team worked around the spatial limitations and arranged the stock in different ways in an effort to find the most appropriate solution. The small room for 
sterile consumables was arranged and rearranged many times, with different shelving configurations, until the arrangement that allowed easiest access was found. Colourcoded labels were used for the consumables to assist with fast and correct identification. The consumable stock was separated from the consignment stock to simplify the reordering process and all cardiopulmonary bypass supplies were stored in close proximity to the OR. Finally, a scout nurse trolley was set up in the OR and stocked with extra supplies ensuring a quick and safe scouting process during surgery. Implementing this strategy to overcome this barrier was achieved through planning and persistence.

\section{Financial barriers}

Cardiac surgery is a rapidly growing specialty that uses more expensive technology than many other surgical specialties $^{19}$. According to ANZSCTS guidelines ${ }^{10}$, a cardiac surgery unit should perform a minimum of 200 cases per year and a surgeon should operate on at least 100 patients per year. Trogdon ${ }^{31}$ stated that a surgery service must provide a minimum number of surgeries per year as high surgical activity correlates to improved health outcomes for patients. With the increase in the number of surgeries, the cardiac team gains more expertise and patients feel more confident about presenting to a newly established unit ${ }^{31}$.

The initial Cardiac Surgery Business Case ${ }^{9}$ specified that a minimum requirement of 400 cardiac surgeries should be performed during 2013 and 2014; however, with Queensland Health's activity-based funding, the GCUH cardiac surgery service implementation had a defined budget. This presented a financial barrier during the set-up phase and numerous ways were explored to ensure that surgical activity targets were met. One solution was reducing the number of days patients stayed in hospital by introducing patient admission on the day of surgery for elective cardiac cases - a practice identified as being cost effective ${ }^{32}$. The second solution was to create clear cardiac patient pathways ${ }^{26}$, including all expected fundamentals of care and treatment for every specific cardiac surgical case through specific cardiac procedures and work instruction. These pathways are designed to support patient-centered care, manage economic costs, offer stability in practice, increase standards of care and maintain accurate clinical records ${ }^{26}$.

Another way to decrease costs was to reduce the time patients spent in the ICU after surgery. It is acknowledged that a lengthy ICU admission increases the cost of the service ${ }^{33}$ and can produce bed-block and have negative impacts on cardiac surgery activity $^{34}$. As the cardiac surgery at GCUH had been allocated only two beds in the ICU, maintaining a continuous flow of the daily surgeries can be achieved by ensuring timely patient transfer to the step-down unit. ICU stay is a significant part of the hospital journey for cardiac surgery patients and the length of ICU stay after cardiac surgery is subjected to different clinical and non-clinical factors and may vary between one and seven days ${ }^{34}$. The implementation process encountered various barriers because of the complexity of the specialty. However, the team identified solutions to overcome these barriers, and have now learnt some valuable and transferrable lessons during the implementation process.

\section{Pitfalls and lessons learned}

The cardiac OR is a complex environment where highly trained professionals work collaboratively using complex equipment to treat patients with severe cardiac disease and major co-morbidities. Wahr et al. ${ }^{35 \text { p. } 1139}$ stated that these dedicated professionals are "human and will make errors' and that avoidable mistakes can occur as a result of poor teamwork and communication rather than technical ability, preparation or knowledge. It is essential to build a cohesive team with effective communication skills underpinned by effective team training, shared knowledge, and conflict resolution ${ }^{35}$. These strategies reduce the potential for errors and improve the quality and safety of patient care $^{36}$. The ability to build a cohesive team through a change management process requires good leadership.

\section{Change management necessitates} effective communication and connections. Establishing these and developing the ability to deal with the change while guiding staff through it is a significant leadership skill $^{37}$. Building the cardiac OR team at GCUH was a process spanning about three years, with difficulties in the recruiting process. We came to realise that the best solution was to train existing staff eager to join the cardiac team. From an organisational and management perspective, it is vital to recognise the existing resources and explore how they could be used successfully to implement change ${ }^{13}$. It is important to identify the requirements for establishing a new cardiac OR but it is also crucial to acknowledge the resources that are already present in the health care facility ${ }^{13}$

Involving all key stakeholders in the implementation process is valuable because it allows for different professional perspectives to be gleaned and implemented. Having stakeholders involved from the beginning would help with identifying possible obstacles and implementing suitable approaches to improve the change process ${ }^{17}$. Establishing a new cardiac unit, and especially a suitable 
cardiac OR, should be planned well in advance with the surgical team (the end-users) involved from the beginning so they can spend time with the architects and the project team ${ }^{7}$ as some pitfalls and barriers could be avoided.

Implementing and establishing a new service in a health care organisation is challenging and the complexity of the organisation makes this process even more difficult. A communication plan should be prepared containing all important information in relation to the aims of the change, who will be involved in the process and how the change will affect them ${ }^{13}$. The significance of information and active communication in health care system is well documented ${ }^{38}$.

\section{Conclusion}

Change management processes in health care organisations are difficult and susceptible to failure because of probable barriers such as human resources, structural or funding constraints. Identifying the possible barriers and enablers, understanding and choosing a proper change management theory and targeted planning from the start of the process ensures a positive outcome ${ }^{17}$. At the GCUH, establishing the new cardiac surgery service has been a long and challenging process and several barriers were identified prior to and during the journey.

The cardiac team has overcome these barriers and four years after the first cardiac surgery was performed our service is well established and has performed over 1100 cardiac surgeries. The cardiac unit successfully implemented day-ofsurgery admission for elective cardiac surgery, and ICU admissions after cardiac surgery are mostly under 24 hours, with very few complicated cases requiring longer ICU care.

Following a long recruitment and training process and numerous changes, the cardiac surgery service has three surgeons, three cardiac registrars and a resident, nine instrument/circulating registered nurses, eight cardiac anaesthetists and medical perfusionists and nine cardiac anesthetic registered nurses. Teamwork, effective communication between all stakeholders, patient- 
focused care and acceptance of change led to establishing a successful service that offers high quality care.

\section{References}

1. Yacoub M, Eluindy A, Afifi A, Yacoub L, Wright G. Taking cardiac surgery to the people. J Cardiovasc Transl Res 2014;7(9):797-802.

2. Clune SJ, Blackford J, Murphy M. Management of the acute cardiac patient in the Australian rural setting: A 12-month retrospective study. Aust Crit Care 2014;27(1):11-16

3. Queensland Health. Cardiac services [Internet]. Brisbane: Queensland Health; 2016 [cited 2017 November 15]. Available from: www.health.qld.gov.au/__data/assets/ pdf_file/0022/444271/cscf-cardiac.pdf.

4. Bojar RM. Manual of perioperative care in adult cardiac surgery. $5^{\text {th }}$ ed. West Sussex, UK: Wiley-Blackwell, 2011.

5. Hardikar A, Skillington P, Shardey G, Smith J. Guidelines for the establishment of an adult cardiac surgery unit (CSU). Heart Lung Circ 2013;22(9):699-702.

6. Pagano D, Kappetein AP, Sousa-Uva M, Beyersdorf F, Klautz R, Mohr F et al. EACTS clinical statement: Guidance for the provision of adult cardiac surgery. Eur J Cardiothorac Surg 2016;50(6):1006-1009.

7. Mehta Y, Bhatia YP. Establishing a new cardiac surgical unit: Challenges and solutions. Ann Card Anaesth 2010;13(3):192-195.

8. Queensland Health. Healthwaves+ Cardiac surgery commences [Internet]. Brisbane: Queensland Health; 2013 [cited 2017 November 16]. Available from: www. goldcoast.health.qld.gov.au/about-us/ publications/healthwaves? page=4.

9. Gold Coast Hospital and Health Services (GCHHS). Cardiac surgery business case (adult cardiac services). Brisbane: Queensland Health, 2013.

10. Australian and New Zealand Society of Cardiac and Thoracic Surgeons (ANZSCTS). Guidelines for the establishment of an adult cardiac surgery unit (CSU) [Internet]. Sydney: ANZSCTS; 2014 [cited 2017 November 15 November]. Available from: anzscts.org/ anzscts-guidelines-for-establishment-ofan-adult-cardiac-surgery-unit

11. Stamp N, Grangert E, Larbalestier R. Modern cardiac surgery: The future of cardiac surgery in Australia. A N Z J Surg 2017;87:661-664

12. Parissis H. Cardiac surgery: what the future holds? J Cardiothorac Surg 2011;6(93):1-3.
13. Allen B. Effective design, implementation and management of change in health care. Nurs Stand 2016:31(3):58-71.

14. Mitchell G. Selecting the best theory to implement planned change. Nurs Manag 2013:20(1):32-37.

15. Kumah E, Ankomah SE, Antwi F. The role of first-line managers in health care change management: A Ghanaian context. IJBST 2016;6(3):20-33.

16. Weiner BJ. A theory of organizational readiness for change. Implement Sci 2009;4(67):1-9.

17. McGreevy J. Implementing culture change in long-term dementia care settings. Nurs Stand 2013;30(19):44-50

18. Shirey MR. Lewin's theory of planned change as a strategic resource. Strategic leadership for organisational change. J Nurs Adm 2013:43(2):69-72.

19. Walton NA, Martin DK, Peter EH, Pringle DM, Singer PA. Priority setting and cardiac surgery: A qualitative case study. Health Policy 2007;80(3):444-458.

20. Kang E, Gillespie BM, Massey D. What are the non-technical skills used by scrub nurses? An integrated review. ACORN 2014;27(4):16-25.

21. Brasher PA, McClelland KH, Denehy L, Story I. Does removal of deep breathing exercises from a physiotherapy program including pre-operative education and early mobilisation after cardiac surgery alter patient outcomes? Aust J Physiother 2003;49(3):165-173.

22. Cebeci F, Celik SS. Discharge training and counselling increase self-care ability and reduce post discharge problems in CABG patients. J Clin Nurs 2008;17(3):412-420

23. Atik FA, Garcia MF, Santos LM, Chaves RB, Faber CN, Corso RB et al. Results of the establishment of an organisational model in cardiovascular surgery service. Rev Bras Cir Cardiovasc 2009;24(2):116-125

24. Queensland Health. Gold Coast Health increases health care services [Internet]. Brisbane: Queensland Health; 2017 [updated 2017 September 29; cited 2018 January 5]. Available from: www.goldcoast.health.qld. gov.au/about-us/news/gold-coast-healthincreases-health-care-services.

25. Queensland Health. Gold Coast University Hospital [Internet]. Brisbane: Queensland Health; 2017 [cited 2017 November] 26. Available from: www.goldcoast.health. qld.gov.au/hospitals-and-centres/goldcoastuniversity-hospital.

26. De Luc K. Care pathways: An evaluation of their effectiveness. J Adv Nurs 2000;32(2):485-496.
27. Kabene SM, Orchard C, Howard JM, Soriano MA, Leduc R. The importance of human resources management in health care: A global context. Hum Resour Health 2006;2(20):1-17.

28. Edmondson AC. Speaking up in the operating room: How team leaders promote learning in interdisciplinary action teams. J Manage Stud 2003;40(6):1419-1452.

29. Fraisse A, Le Bel S, Mas B, Macrae D. Paediatric cardiac intensive care unit: Current setting and organization in 2010. Arch Cardiovasc Dis 2010:103(10):546-551.

30. Australian College of Operating Room Nurses (ACORN). Standards for Perioperative Nursing in Australia. 14 $4^{\text {th }}$ ed. Adelaide: ACORN, 2016.

31. Trogdon JG. Demand for and regulation of cardiac services. Int Econ Rev 2009;50(4):1183-1204.

32. Silvay G, Goldberg A, Gutsche JT, Augoustides JGT. Same day admission for elective cardiac surgery: How to improve outcome with satisfaction and decrease expenses. J Anesth 2016:30:444-448.

33. Kern H, Kox WJ. Impact of standard procedure and clinical standards on cost-effectiveness and intensive care unit performance in adult patients after cardiac surgery. Intensive Care Med 1999;25(12):13671373.

34. Almashrafi A, Elmontsri M, Aylin P. Systematic review of factors influencing length of stay in ICU after adult cardiac surgery. BMC Health Serv Res 2016;16(318):1-12.

35. Wahr JA, Prager RL, Abernathy JH, Martinez EA, Salas E, Seifert PC et al. Patient safety in the cardiac operating room: Human factors and teamwork: A scientific statement from the American Heart Association. Circulation 2013;128(10):1139-1169.

36. De Leval MR, Carthey J, Wright DJ, Farewell VT, Reason JT. Human factors and cardiac surgery: A multicenter study. I Thorac Cardiovasc Surg 2000; 119(4/1):661-672.

37. Macphee M, Suryaprakash N. First-line nurse leaders' health-care change management initiatives. J Nurs Manag 2012;20(2):249-259.

38. Dahl AB, Abdallah AB, Maniar H, Avidan MS, Bollini ML, Patterson GA et al. Building a collaborative culture in cardiothoracic operating rooms: Pre- and postintervention study protocol evaluation of the implementation of teamSTEPPS training and the impact of perceived psychological safety. BMJ Open 2017;7(9):1-6. 